\title{
Histologically confirmed nonbacterial thrombotic endocarditis in a febrile leukemic patient
}

Eun Gyo Jeong, Mi-Hyang Jung, and Ho-Joong Youn

Cardiovascular Center, College of Medicine, Seoul St. Mary's Hospital, The Catholic University of Korea, Seoul, Korea
Received: April 20, 2016

Revised : May 12, 2016

Accepted: May 23, 2016

\section{Correspondence to}

Mi-Hyang Jung, M.D.

Cardiovascular Center, College of Medicine, Seoul St. Mary's Hospital, The Catholic University of Korea, 222 Banpo-daero, Seocho-gu, Seoul 06591, Korea

Tel: +82-2-2258-1128

Fax: +82-2-2258-1142

E-mail: floria0515@gmail.com
To the Editor,

A 53-year-old man was admitted because of fever and right upper quadrant pain that had persisted for 5 days. He had no previously known disease. His initial temperature was $38.3^{\circ} \mathrm{C}$, and a grade III diastolic murmur was auscultated at the right upper sternal border. The initial laboratory findings were suggestive of acute promyelocytic leukemia and a disseminated intravascular coagulation (DIC) state was suspected. The levels of the inflammatory markers such as the high-sensitivity C-reactive protein $(9.6 \mathrm{mg} / \mathrm{dL})$ and pro- calcitonin (0.774 ng/mL) were slightly elevated. Contrast-enhanced abdominal computed tomography revealed multiple embolic infarctions in the spleen and kidney. Transthoracic echocardiography revealed an echogenic mass attached to the aortic valve (AV) (Fig. 1A), and Doppler studies revealed moderate to severe aortic regurgitation (AR) (Fig. $1 \mathrm{~B}$ and ${ }_{1} \mathrm{C}$ ). Further evaluation with transesophageal echocardiography revealed low echogenic masses attached to the tip of the right coronary cusp and noncoronary cusp (Fig. 1D), resulting in moderate to severe AR.
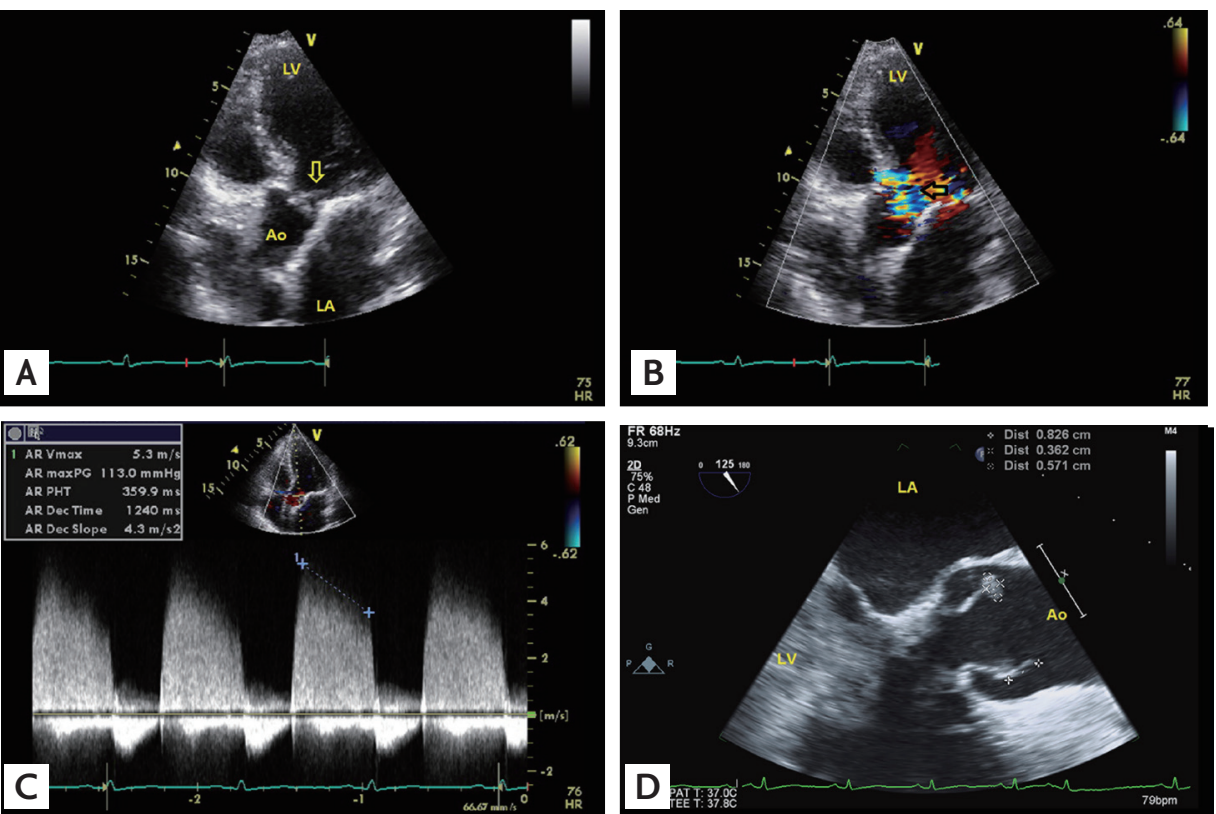

Figure 1. (A) Transthoracic echocardiogram showing an echogenic mass (arrow) attached to the aortic valve, $(\mathrm{B}, \mathrm{C})$ resulting in moderate to severe aortic regurgitation. (D) Transesophageal echocardiogram showing an echogenic mass attached to both the right and non-coronary cusps of the aortic valve. LV, left ventricle; Ao, aorta; LA, left atrium. 


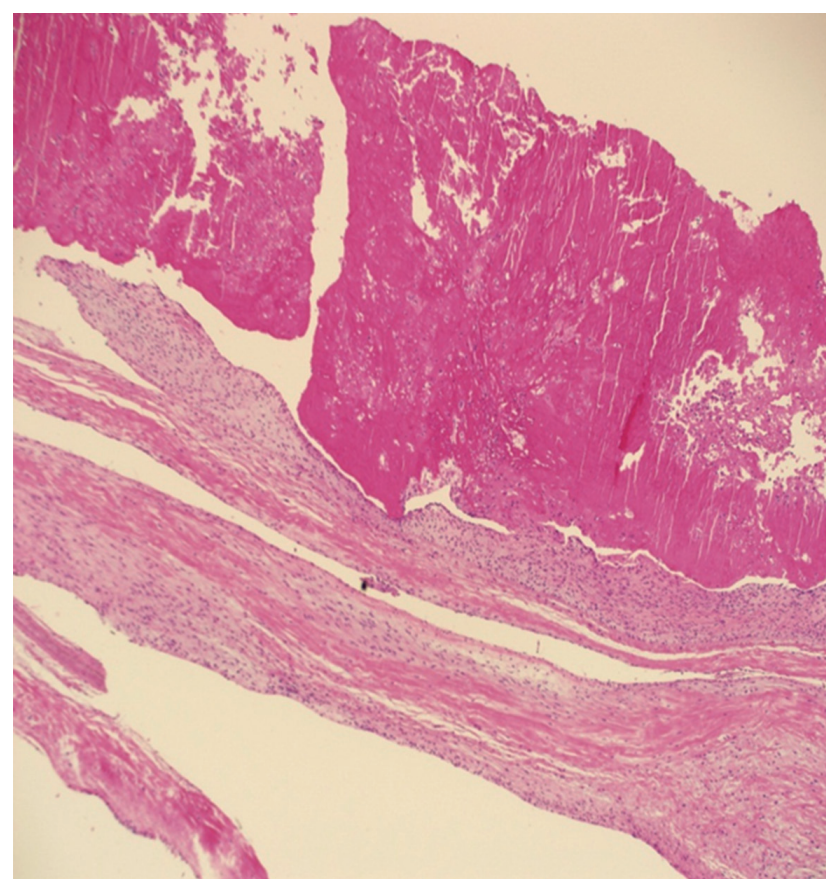

Figure 2. Pathologic finding showing vegetation attached to the valve endothelium, mainly composed of a fibrin clot with few inflammatory cells, consistent with nonbacterial thrombotic endocarditis $(\mathrm{H} \& \mathrm{E}, \times 100)$. The lack of an inflammatory response to the valve structure was an important clue to the final diagnosis.

Based on the aforementioned findings, he was highly suspected as having infective endocarditis involving the AV. Because he was in a neutropenic state and had fever, we started intravenous antibiotics (vancomycin and gentamicin) immediately after obtaining blood cultures. Despite using broad-spectrum antibiotics, follow-up echocardiography performed after 1 week revealed no shrinkage of the vegetations and persistent moderate to severe AR. Moreover, recurrent fever was noted. Therefore, the possibility of local uncontrolled infection could not be ruled out. Although blood sample cultures yielded negative results, the patient was scheduled to undergo $\mathrm{AV}$ replacement and removal of the infection focus, given that the possibility of culture-negative infective endocarditis by fastidious organisms still existed in this clinical setting (febrile immune compromised patient who is scheduled to receive high dose chemotherapy). Moreover, the patient had developed thromboembolic events. The operative findings were consistent with the preoperative findings, and his postoperative course was uneventful. However, the final pathological examination showed that the vegetation adhered to the valve endothelium was mainly composed of fibrin and had few inflammatory cells (Fig. 2). The most important pathologic finding was a lack of inflammatory response. Therefore, he was diagnosed with nonbacterial thrombotic endocarditis (NBTE), and chemotherapy (arsenic trioxide combined with all-transretinoic acid) was started. He continued to do well clinically without fever or dyspnea.

Clinical diagnosis is quite tricky when vegetations are combined with fever in an autoimmune or myeloproliferative disease because of the vegetation sites being identical to those in infective endocarditis [1]. Furthermore, fever is a common presentation of diseases that are of either metabolic or infective etiologies [1]. Additionally, infective endocarditis by blood culture-negative fastidious organisms should also be considered [2,3]. However, as in the present case, valve replacement could be considered in patients with combined valve dysfunction, and histological evaluation of the resected valve tissue may facilitate the diagnosis [2].

In conclusion, despite the low incidence of NBTE, when cultures yield negative results and highly thrombogenic patients are not responsive to empirical antibiotic treatment, the possibility of NBTE should be considered.

Keywords: Endocarditis; Leukemia; Endocarditis, non-infective

\section{Conflict of interest}

No potential conflict of interest relevant to this article was reported.

\section{REFERENCES}

1. Reisner SA, Brenner B, Haim N, Edoute Y, Markiewicz W. Echocardiography in nonbacterial thrombotic endocarditis: from autopsy to clinical entity. J Am Soc Echocardiogr 2000;13:876-881.

2. Katsouli A, Massad MG. Current issues in the diagnosis and management of blood culture-negative infective and non-infective endocarditis. Ann Thorac Surg 2013;95:1467-1474.

3. Tattevin P, Watt G, Revest M, Arvieux C, Fournier PE. Update on blood culture-negative endocarditis. Med Mal Infect 2015;45:1-8. 